\title{
Home based physical activity intervention programme in war-torn country like Iraq
}

\begin{abstract}
The unsuitable conditions as the result of a decade of war had made it difficult to raise the level of physical activity among the youth in Iraq. Lack of awareness, as well as misconceptions about diets and the concept of physical activity among young people and the society in general especially pertaining women involvement in sports had made this matter worsen. Hence, designing home-based intervention program to enhance the physical activity level among the sedentary undergraduate female students in Iraq is crucial. The home based intervention program consists of simple exercises to be carried out at home and some nutrition information on balance diet. Forty-four sedentary undergraduate female students aged 18-22 years old were selected as subjects in this study. They were randomly selected from Northern Region of Iraq. The subjects completed a 12-weeks home based intervention program that combining simple exercises and diet information. Their daily physical activity level was measured using a pedometer. The results showed that there were a significant difference between the steps counts from pre-test to post-test1 (6-weeks) and pre-test to posttest2 (12-weeks) $(\mathrm{p}<0.001)$ in the experimental group. The mean steps increased by 6825.73 steps from pre-test to post-test1, and 9007.71 steps from pre-test to post-test2. Additionally, the results of these two groups were different in test (time) pre, post 1 and post $2(\mathrm{~F}(2,84)=$ $713.00, \mathrm{P}<0.05, \cdot 2=.944)$. Based on these results, it was concluded the 12 -weeks home based intervention program was effective in enhancing physical activity level among sedentary undergraduate female students in Iraq.
\end{abstract}

Keyword: Home based intervention program; Physical activity; Undergraduate; Female 